\title{
Sources of hospital-level variation in functional outcome after acute ischemic stroke: a multicenter retrospective cohort study
}

\author{
Runhua Zhang ${ }^{1,2,3}$, Gaifen Liu ${ }^{2,3}$, Yuesong Pan ${ }^{2,3}$, Yong Jiang ${ }^{2,3}$, Xinying Huang ${ }^{2,3}$, Xia Meng ${ }^{2,3}$, \\ Yongjun Wang ${ }^{2,3}$, Maigeng Zhou ${ }^{1}$ \\ ${ }^{1}$ National Center for Chronic and Noncommunicable Disease Control and Prevention, Chinese Center for Disease Control and Prevention, Beijing, \\ China; ${ }^{2}$ Department of Neurology, Beijing Tiantan Hospital, Capital Medical University, Beijing, China; ${ }^{3}$ China National Clinical Research Center \\ for Neurological Diseases, Beijing, China \\ Contributions: (I) Conception and design: R Zhang, M Zhou, Y Wang; (II) Administrative support: G Liu, X Meng; (III) Provision of study materials \\ or patients: Y Wang; (IV) Collection and assembly of data: Y Jiang, X Huang; (V) Data analysis and interpretation: R Zhang, G Liu, Y Pan; (VI) \\ Manuscript writing: All authors; (VII) Final approval of manuscript: All authors. \\ Correspondence to: Maigeng Zhou, PhD. National Center for Chronic and Noncommunicable Disease Control and Prevention, Chinese Center for \\ Disease Control and Prevention, No. 27 Nanwei Road, Xicheng District, Beijing, China. Email: maigengzhou@126.com.
}

Backgroundz Functional recovery is an important clinical outcome for patients with stroke. Hospital-level
variation occurs in functional outcome after stroke. However, the extent to which patient characteristics,
hospital characteristics, process of care, and medication persistence contribute to the hospital variation in
poor functional outcome after ischemic stroke is unknown in China.
Methods: We retrospectively analyzed patients enrolled in the Third China National Stroke Registry
from 2015 to 2018 . The outcome was poor functional outcome (modified Rankin Scale score of 3-6) at
3 months and 1 year. We used the median odds ratio to quantify the hospital-level variation in rates of poor
functional outcome. A series of hierarchical logistic models were constructed to assess the extent to which
patient characteristics, hospital characteristics, process of care, and medication persistence contributed to the
hospital variation. Results: A total of 13,218 patients with acute ischemic stroke from 159 hospitals were included. The riskadjusted rates of the poor functional outcome at 3 months and 1 year after ischemic stroke varied across hospitals, ranging from $6.0 \%$ to $26.0 \%$ and $6.8 \%$ to $24.0 \%$, respectively. The median odds ratio was 1.78 at 3 months and 1.67 at 1 year. Hierarchical logistic models indicated that the patient characteristics, hospital characteristics, process of care, and the medication persistence explained $27.5 \%, 7.0 \%, 6.5 \%$, and $5.0 \%$ of the variation at 1 year. Altogether, $36.8 \%$ and $46.1 \%$ of the hospital variation in 3 -month and 1 -year poor functional outcome, respectively, was explained.

Conclusions: There was a wide variation in rates of functional outcomes across hospitals. Approximately half of the variation at 1 year can be explained by patient characteristics, hospital characteristics, process of care, and medication persistence. Approximately $11.5 \%$ of the variation can be modified through improvements in the process of care and medication persistence. More studies are needed to explore the contribution of post-discharge care in the future.

Keywords: Variation; outcome; ischemic stroke; contribution

Submitted Jul 08, 2021. Accepted for publication Sep 07, 2021.

doi: 10.21037/apm-21-1860

View this article at: https://dx.doi.org/10.21037/apm-21-1860 


\section{Introduction}

Stroke is one of the leading causes of mortality and disability worldwide (1). Stroke patients are more likely to be left with disability than to die of stroke (2). It was estimated that there were 80.1 million stroke survivors globally in 2006, with 24 million stroke survivors in China $(3,4)$. Improving the outcome for patients after stroke is the main objective for the management of stroke. In the past decades, substantial advancements in stroke care have been made, such as intravenous thrombolysis, endovascular clot retrieval $(5,6)$, and antiplatelet and lipid-lowering therapies $(7,8)$. These have been incorporated into stroke patient management guidelines and are widely employed in clinical practice to improve the quality of care. To evaluate and monitor the quality of care, some indicators were proposed, such as antiplatelet medication within 48 hours after stroke onset, antiplatelet medication at discharge, early rehabilitation, screening for swallowing disorders, and so on (9). The outcome indicators were often used to profile the hospital performance, such as 30-day mortality, readmission, and functional recovery (2).

Functional status, which is more sensitive than vital status, may reflect the process of care or stroke-related intervention. Additionally, functional impairment is a common sequela of stroke and functional status, which is a patient-center metric, and is of significance to patients and their families (2). A better understanding of the hospital variation in functional outcome can help to assess the quality of care and guide the measures to reduce the variations. Although previous studies have found the variation in functional outcomes if the patients were treated in different hospitals, it was uncertain about the sources of the hospital variation $(10,11)$. This hospital-level variation in functional outcome may result from the patient characteristics, hospital characteristics, process of care, medication persistence, or chance. However, the extent to which these sources contribute to the hospital variation in functional outcomes for patients with acute ischemic stroke is unclear. Using data from national perspective stroke registry, this study aimed to examine whether hospital variation in functional outcomes exists for patients with acute ischemic stroke in China and to explore the extent to which patient characteristics, hospital characteristics, process of care, and medication persistence contribute to this variation.

We present the following article in accordance with the STROBE reporting checklist (available at https://dx.doi. org/10.21037/apm-21-1860).

\section{Methods}

\section{Data source}

We used the data from the Third China National Stroke Registry (CNSR-III) study. The details of the CNSRIII are published elsewhere (12). Briefly, CNSR-III is a multicenter prospective nationwide stroke registry study. Overall, 15,166 patients with acute ischemic stroke and transient ischemic attack were consecutively included from 201 hospitals in China from August 2015 to March 2018. Patients aged older than 18 years and within 7 days of the symptom onset were recruited. According to the World Health Organization criteria, stroke is defined as "rapidly developing clinical signs of focal (or global) disturbance of cerebral function, lasting more than 24 hours or leading to death, with no apparent cause other than that of vascular origin (13)". In all patients, acute ischemic stroke was confirmed by magnetic resonance imaging or brain computed tomography. Trained research coordinators collected the baseline data through an electronic data capture system. Patient demographics, medical history, risk factors, in-hospital therapies, the National Institutes of Health Stroke Scale (NIHSS) score, and modified Rankin Scale (mRS) score were extracted from the medical records or interview. Follow-ups were performed at 3, 6, and 12 months by face-to-face or telephone interview. The information on functional dependence, cardiovascular events, medication, and risk factor controlling were recorded during the follow-up. The study was conducted in accordance with the Declaration of Helsinki (as revised in 2013). The CNSR-III study was approved by the institutional review board of Beijing Tiantan Hospital (NO. KY2015-001-01) and informed consent was taken from all the patients.

\section{Outcome and study population}

The outcome of the present study was poor functional outcome defined as mRS score of 3-6 at 3 months and 1 year $(10,11)$. In this study, we included all patients with acute ischemic stroke. We excluded those missing related covariates in the adjusted model as described below $(\mathrm{n}=137)$. To obtain a reliable estimation of 1 -year functional outcomes, we also excluded patients admitted to hospitals with $<20$ patients $(\mathrm{n}=413)$. The patients lost at 3 months or 1 year were also excluded $(n=378)$. Finally, our analytical cohort in this study comprised of 13,218 patients from 159 hospitals. The details of the patient inclusion are described 


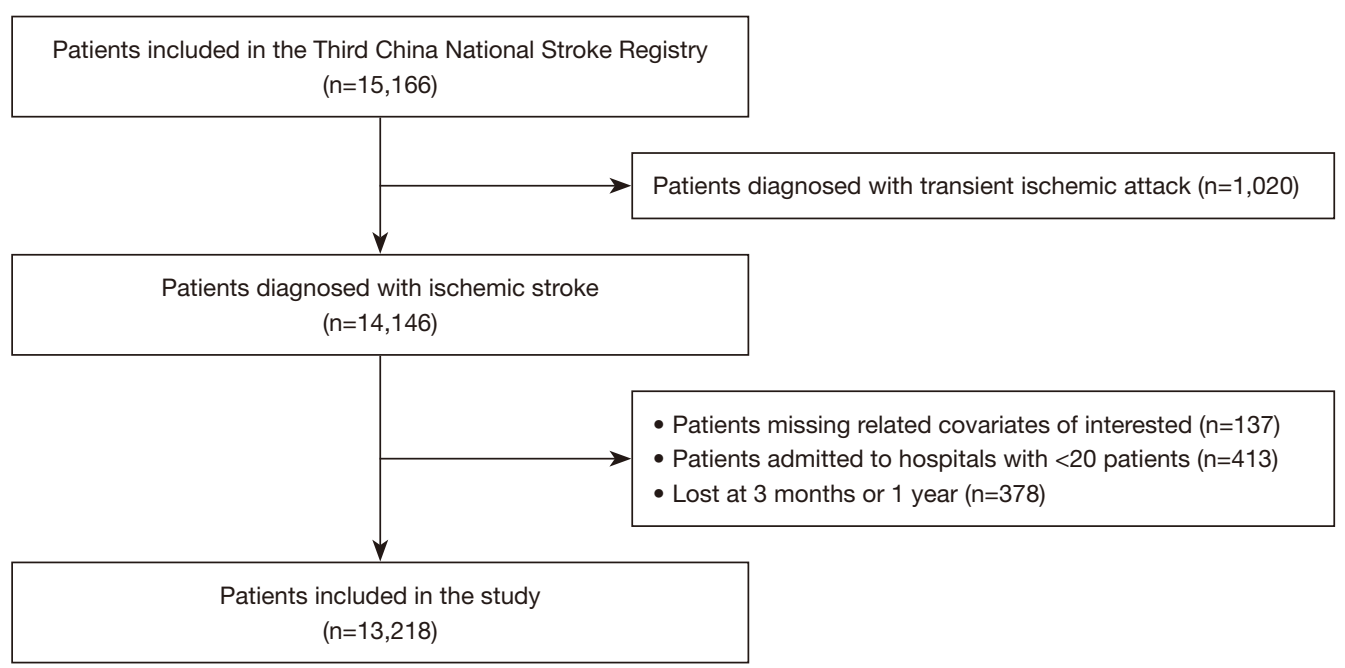

Figure 1 Flowchart of patient selection.

in Figure 1.

\section{Covariates}

To adjust for the patient characteristics, we chose a list of variables based on the literature and clinical relevance. The variables were adjusted as follows: age, sex, stroke severity (NIHSS score, subdivided into four risk categories based on the quartiles), stroke etiology (five categories based on Trial of Org 10172 in Acute Stroke Treatment, TOAST: large artery atherosclerosis, cardiogenic embolism, small artery occlusion, stroke of another determined cause, stroke of an undetermined cause), hypertension, hyperlipidemia, atrial fibrillation, diabetes, coronary heart disease, current smoking, cancer, renal dialysis, and the pre-stroke dependency (independent with $\mathrm{mRS}$ score $\leq 2$, dependent with $\mathrm{mRS}$ score $>2$ ).

Hospital characteristics assessed in this study included the status of academic, geographic region (east, central, west), and stroke unit. All these data were collected from the participating hospitals.

Given that the process of care was another potential source of hospital variation in functional outcomes, we selected nine processes of care that can be applied to the patients with ischemic stroke regarding the national indicator of the quality of care, stroke management guidelines (14), and Get With The Guidelines-Stroke (15): (I) intravenous thrombolysis (recombinant tissue plasminogen activator, rtPA), (II) endovascular therapy (mechanical thrombectomy, MT), (III) in-hospital antiplatelet medicine, (IV) oral anticoagulation for patients with atrial fibrillation, (V) in-hospital medication for lowering low-density lipoprotein cholesterol (LDL-C), (VI) antihypertensive medicine, (VII) hypoglycemic treatment, (VIII) deep vein thrombosis (DVT) prophylaxis, and (IX) the evaluation of limb rehabilitation before discharge.

Additionally, medication persistence after discharge was an important factor for outcomes after acute ischemic stroke $(16,17)$. In this study, we considered the information on medication persistence at 3 months after discharge as another potential source of variation in functional outcomes after stroke. Medication persistence was defined as continuously taking medication from discharge to 3 -month post-discharge. If the patients failed to take any prescribed medications, including antihypertensive agents, hypoglycemic medicine, antiplatelet agents, oral anticoagulation, and lipid-lowering drugs, at 3-month postdischarge, the patient was defined as "non-persistence" as the previous study (18).

\section{Statistical analyses}

For descriptive purposes, we used the quartiles of riskadjusted hospital-level rates of poor functional outcome at 1 year (described as model 1 below) to categorize the hospitals into four groups (Q1-Q4). Patients' characteristics and hospital characteristics were described using means and standard deviation or medians and interquartile ranges 
(IQRs) for continuous variables and percentages for categorical variables. To test the linear trend of baseline characteristics of patients and hospitals by groups, we used Cochran-Mantel-Haenszel non-zero correlation tests for continuous variables and Cochran-Mantel-Haenszel row mean scores for categorical variables. We used hierarchical logistic models to investigate the variations in poor functional outcome at 3 month and 1 year with a random intercept to account for inter-hospital correlation. To facilitate the interpretation of the variability, we used the median odds ratio (MOR) as per previous study (19), which quantified the average increased risk of an identical patient moving randomly from a hospital with a lower risk to another hospital with a higher risk.

A series of hierarchical logistical models were constructed to assess the degree to which patient characteristics, hospital characteristics, process of care, and medication persistence contributed to the variation in hospital-level rates of poor functional outcome. First, the null model (Model 0) was developed with only hospital-specific random intercepts and no covariates included. In the view of potential sources of hospital-level rates of poor functional outcome, the subsequent models were constructed. In Model 1, we added the patient characteristics to adjust for the case mix. Based on Model 1, the risk-adjusted rates for each hospital were estimated using predicted versus expected ratios multiplied by the overall unadjusted rates of poor functional outcome (2). The included hospitals were classified into four groups based on the quartiles of risk-adjusted rates to describe the baseline characteristics. Model 2 consisted of the covariates in Model 1 plus hospital characteristics. Model 3 consisted of the covariates in Model 2 and the process of care, including MT and rtPA, the antiplatelet therapy, oral anticoagulation, lipid-lowering therapy, antihypertensive medicine, hypoglycemic treatment, DVT prophylaxis, and the evaluation of limb rehabilitation. Model 4 consisted of the covariates in Model 3 plus the allor-none medication persistence. We used the proportional change in variance (PCV) to quantify the extent to which patient characteristics, hospital characteristics, process of care, and medication persistence contributed to the hospital-level variation in rates of poor functional outcome. The PCV equation is as follows: $P C V=\frac{V_{A}-V_{B}}{V_{A}}$, where $V_{A}$ is the variance of the initial model and $V_{B}$ is the variance of

the subsequent model with additional covariates $(20,21)$. The PCV indicates the proportion of hospital variation explained by the additional covariates.

Additionally, several sensitivity analyses were conducted. First, we performed a sensitivity analysis by including all patients in all hospitals and patients in hospitals with $>50$ patients included. Second, we used multiple imputation method to reduce the bias caused by missing data. Third, the composite persistence defined as percentage of discharge medication classes that subjects reported taking at 3 months was also used to do a sensitivity analysis (22).

A two-sided $\mathrm{P}$ value $<0.05$ was considered statistically significant. SAS software version 9.4 (SAS Institute Inc, Cary, NC) was used for all analyses.

\section{Results}

A total of 13,218 patients with acute ischemic stroke from 159 hospitals from August 2015 to March 2018 were included in the study. Figure 1 displays the inclusion and exclusion criteria. The mean age of the patients was $62.2 \pm 11.2$ years, and $31.3 \%$ were female. Hypertension presented in $62.7 \%$ of included patients. The median NIHSS score was 3 (IQR, 2-6). Of these patients, 1,878 $(14.2 \%)$ and $1,780(13.5 \%)$ were functionally dependent or dead at 3 months and 1 year, respectively.

The characteristics of the patients and hospitals by quartiles of risk-adjusted rates of poor functional outcome are listed in Table 1. Based on the quartile of risk-adjusted rates, the hospitals were categorized into four groups ( $\leq 11.1 \%$ for Q1, $11.2 \%$ to $13.2 \%$ for Q2, $13.3 \%$ to $16.4 \%$ for Q3, >16.4\% for Q4). Patients presenting to hospitals with high risk-adjusted rates were more likely to be older, with a higher prevalence of hypertension, atrial fibrillation, and large artery atherosclerosis. No difference was noted in other comorbidities. Compared with Q4, the patients presenting to hospitals with a low risk-adjusted rate of poor functional outcome tended to be with pre-stroke dependence $(\mathrm{P}<0.001)$. Of the 159 hospitals, 124 (78\%) provided organized stroke unit care and $122(76.7 \%)$ were academic hospitals. More than half of the participating hospitals were located in the east of China.

Table 2 shows the process of care patients received. Overall, $8.8 \%$ of the patients received rtPA. The proportion of MT was low in all groups, and we observed no significant difference in the proportions of MT between groups $(\mathrm{P}=0.746)$. A relatively high proportion of patients received antiplatelet and LDL-C-lowering medication during hospitalization. There were $10.3 \%$ of patients received oral anticoagulation. The difference 
Table 1 Patient and hospital characteristics by quartiles of hospital level rates of poor functional outcome at 1 year

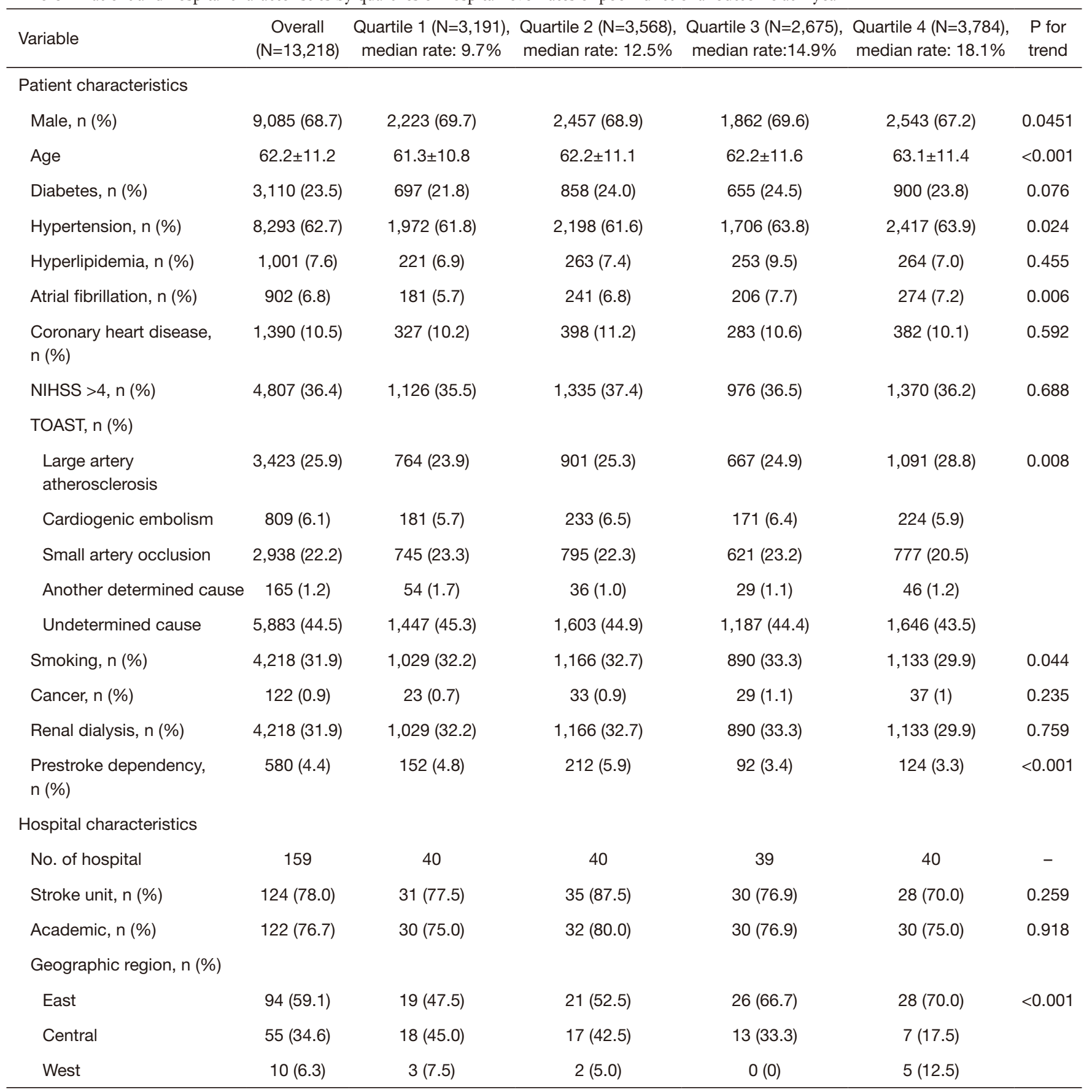

NIHSS, National Institutes of Health Stroke Scale; TOAST, Trial of Org 10172 in Acute Stroke Treatment.

of LDL-C-lowering and antihypertension treatment among groups was statistically significant $(\mathrm{P}<0.001)$. Limb rehabilitation was evaluated in $73.4 \%$ of the patients before discharge. Patients presenting to the hospitals with high risk-adjusted rates were more likely to receive DVT prophylaxis. There was a significantly lower prevalence of medication persistence at 3 months in hospitals with high risk-adjusted rates $(\mathrm{P}<0.001)$. 
Table 2 The process of care and medication persistence in hospital by quartiles of hospital level rates of poor functional outcome at 1 year

\begin{tabular}{|c|c|c|c|c|c|c|}
\hline Variable & $\begin{array}{c}\text { Overall } \\
(\mathrm{N}=13,218)\end{array}$ & $\begin{array}{c}\text { Quartile } 1(\mathrm{~N}=3,191), \\
\text { Median rate: } 9.7 \%\end{array}$ & $\begin{array}{l}\text { Quartile } 2(\mathrm{~N}=3,568) \text {, } \\
\text { Median rate: } 12.5 \%\end{array}$ & $\begin{array}{c}\text { Quartile } 3(\mathrm{~N}=2,675) \text {, } \\
\text { Median rate: } 14.9 \%\end{array}$ & $\begin{array}{l}\text { Quartile } 4(\mathrm{~N}=3,784) \text {, } \\
\text { Median rate: } 18.1 \%\end{array}$ & $\begin{array}{l}\mathrm{P} \text { for } \\
\text { trend }\end{array}$ \\
\hline rtPA, n (\%) & $1,158(8.8)$ & $218(6.8)$ & $357(10.0)$ & $334(12.5)$ & $249(6.6)$ & 0.918 \\
\hline $\begin{array}{l}\text { Lowering LDL-C treatment, } \\
\mathrm{n}(\%)\end{array}$ & $12,782(96.7)$ & $3,074(96.3)$ & $3,405(95.4)$ & $2,610(97.6)$ & $3,693(97.6)$ & $<0.001$ \\
\hline Antiplatelet treatment, n (\%) & $12,864(97.3)$ & $3,113(97.6)$ & $3,455(96.8)$ & $2,603(97.3)$ & $3,693(97.6)$ & 0.544 \\
\hline Oral anticoagulation, $\mathrm{n}(\%)$ & $1,357(10.3)$ & $277(8.7)$ & $381(10.7)$ & $360(13.5)$ & $339(9.0)$ & 0.353 \\
\hline $\begin{array}{l}\text { Hypoglycemic treatment, } \\
n(\%)\end{array}$ & $3,390(25.6)$ & $782(24.5)$ & $911(25.5)$ & $707(26.4)$ & $990(26.2)$ & 0.093 \\
\hline $\begin{array}{l}\text { Evaluation of limb } \\
\text { rehabilitation, } n(\%)\end{array}$ & $9,697(73.4)$ & 2,336 (73.2) & $2,532(71.0)$ & $2,111(78.9)$ & $2,718(71.8)$ & 0.518 \\
\hline $\begin{array}{l}\text { Medication persistence, } \\
\mathrm{n}(\%)\end{array}$ & $10,648(80.6)$ & $2,729(85.5)$ & $2,869(80.4)$ & $2,126(79.5)$ & $2,924(77.3)$ & $<0.001$ \\
\hline
\end{tabular}

rtPA, tissue plasminogen activator; LDL-C, low-density lipoprotein cholesterol; DVT, deep vein thrombosis.

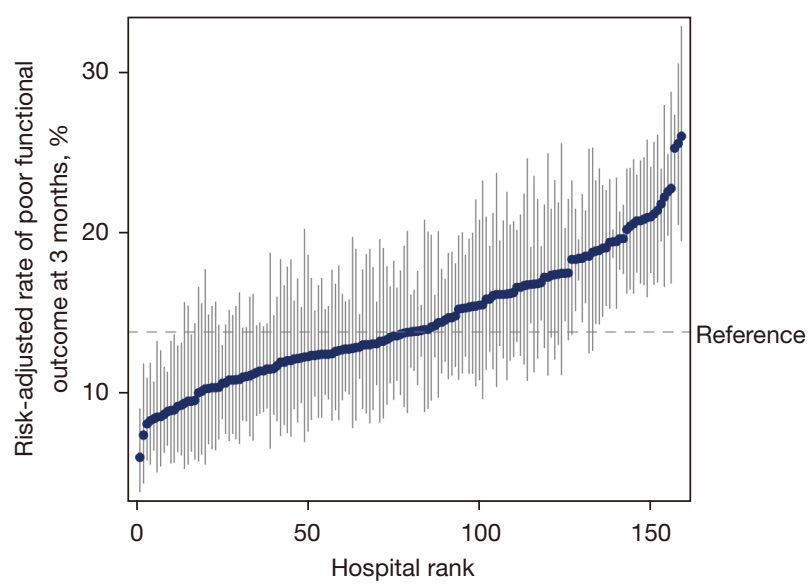

Figure 2 Hospital variability in rates of 3 -month poor functional outcome after acute ischemic stroke. The reference indicates the median risk-adjusted rate of poor functional outcome with a value of $13.8 \%$.

\section{Hospital-level variation in rates of poor functional outcome}

The median hospital-level crude rate of poor functional outcome was $13.2 \%$ (IQR, $8.1 \%$ to $19.4 \%$ ) at 3 months and $12.7 \%$ (IQR, $7.8 \%$ to $18.5 \%$ ) at 1 year. When adjusted for the patient characteristics, the median risk-adjusted rate of poor functional outcome was $13.8 \%$ (IQR, $11.5 \%$ to $17.2 \%$ ) at 3 months and $13.2 \%$ IQR, $11.1 \%$ to $16.4 \%$ ) at 1 year. We ranked all hospitals based on the risk-adjusted rate of poor functional outcome (Figures 2,3). The risk-adjusted rates of poor functional outcome at 3 months and 1 year after ischemic stroke varied widely across hospitals, ranging from $6.0 \%$ to $26.0 \%$ and $6.8 \%$ to $24.0 \%$, respectively. There was an approximately two-fold difference in the risk-adjusted rates of poor functional outcome between the 10th (9.4\%) and 90th $(18.4 \%)$ percentile hospital at 1 year. The MOR was 1.78 at 3 months and 1.67 at 1 year, which suggested that if an identical patient moved from a lower-risk hospital to a higher-risk hospital, the increased risk of poor functional outcome (in median) was $78 \%$ at 3 months and $67 \%$ at 1 year.

\section{Sources of hospital-level variation in rates of poor functional outcome}

We constructed a series of multilevel logistic models to estimate the extent to which patient characteristics, hospital characteristics, process of care during hospitalization, and medication persistence contributed to the hospital-level 
variation in rates of poor functional outcome. Compared to the unadjusted model, the PCV reduction in Model 1 was $32.0 \%$ and $27.5 \%$, suggesting that the patient characteristics can explain $32.0 \%$ and $27.5 \%$ of the hospital variation in rates of poor functional outcome at 3 months and 1 year, respectively. When incorporating hospital characteristics, process of care in hospitalization, and medication persistence in Model 4, overall, $36.8 \%$ and $46.1 \%$ of the variation can be explained.

The incremental PCV is shown in Table 3. The patient characteristics explained the larger proportion of the variation. For the poor functional outcome at 3 months and 1 year, the hospital characteristics accounted for $2.6 \%$ and $7.0 \%$ of the hospital-level variation. When adjusting the process of care and medication persistence, additional $0.8 \%$ and $1.4 \%$ of the variation for 3 -month functional

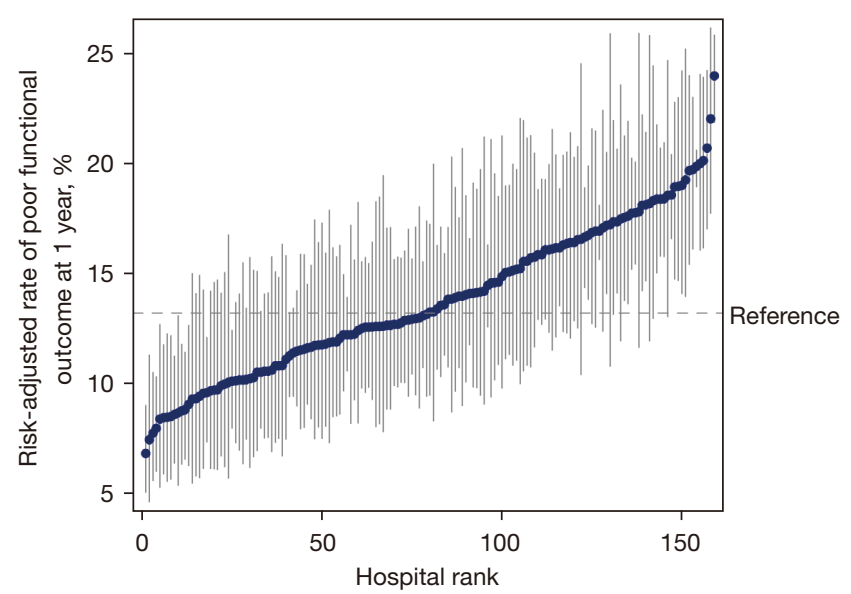

Figure 3 Hospital variability in rates of 1-year poor functional outcome after acute ischemic stroke. The reference indicates the median risk-adjusted rate of poor functional outcome with a value of $13.2 \%$. outcome and $6.5 \%$ and $5.0 \%$ for 1-year functional outcome were explained. The extent to which patient characteristics, hospital characteristics, process of care, and medication persistence contributed to the variation changed little in the sensitivity analyses (data not shown).

\section{Discussion}

In this large national registry of stroke, we found that there was a significant hospital-level variation in rates of poor functional outcome. If an identical patient with ischemic stroke moved from a hospital with a lower risk of poor functional outcome to a hospital with higher risk, the risk of poor functional outcome at 3 months and 1 year increased by $78 \%$ and $67 \%$ respectively, in the median. This hospital variation can be attributed to the patient characteristics, hospital characteristics, process of care patient received, and medication persistence; together, they explained $36.8 \%$ and $46.1 \%$ of the variation at 3 months and 1 year. The remaining more than half of the hospital variation in functional outcomes remains unexplained.

This study demonstrated that a wide variation for functional outcomes existed in patients with acute ischemic stroke, despite age, the severity of stroke, and comorbidities, which were adjusted for. This was consistent with the results of previous studies. The Netherlands Stroke Survey in 10 centers also revealed variation in poor functional outcome at 1 year (25th percentile to 75 th percentile, $37 \%$ to $75 \%$ ) (11). Similarly, a study of 82 US hospitals with 2083 ischemic stroke patients indicated significant variation in functional dependence or death, ranging from $0 \%$ to $67 \%$ by hospitals (10). We note that compared with their studies, the rate of poor functional outcome in our study was lower. This may be because most of the patients in our study had mild strokes with a median NIHSS score of 3

Table 3 Contribution of patient characteristics, hospital characteristics, process of care, and medication persistence to hospital-level variation in rates of poor functional outcome after acute ischemic stroke

\begin{tabular}{|c|c|c|c|c|}
\hline Model & \multicolumn{2}{|c|}{ Poor functional outcome at 3 months } & \multicolumn{2}{|c|}{ Poor functional outcome at 1 year } \\
\hline Model 2: Model 1 + Hospital characteristics & $34.6 \%$ & $2.6 \%$ & $34.5 \%$ & $7.0 \%$ \\
\hline Model 3: Model 2 + Process of care & $35.4 \%$ & $0.8 \%$ & $41.1 \%$ & $6.5 \%$ \\
\hline
\end{tabular}

$\mathrm{PCV}$, proportional change in variance. 
(IQR, 2-6) and were younger in our cohort. Although the rate of poor functional outcome at 1 year was lower than those of the two previous studies, all indicated the hospitallevel variation in functional outcome after adjusting for the severity of stroke, age, and potential covariates.

In this study, we found that the baseline characteristics of patients were the most crucial factor in the variation in functional outcome among other identified potential sources. Patients' characteristics, including age, the severity of the stroke, preadmission dependence, and comorbidities, were significantly associated with the outcome after ischemic stroke. Based on the characteristics of patients, some prognostic models were developed to predict short-term or long-term outcomes after ischemic stroke $(23,24)$. The prognostic model showed good discrimination and calibration in the external validation $(25,26)$, which indicated the paramount effect of the characteristics of patients on functional outcomes. When profiling the performance of the hospital, it is necessary to adjust the heterogeneity of patients, especially in observational studies (2). Risk-adjusted functional status was recommended to be used for controlling the case-mix effect and to homogenize the hospitals (2). The patient characteristics contributed more than one-fourth of the variation in functional outcome, suggesting that intensive measures should be taken to decrease the prevalence of comorbidities and ultimately reduce the burden of stroke disability or mortality.

It was reasonable that hospital characteristics may be another source of hospital variation in functional outcomes. However, a small percentage of variation was explained by the hospital characteristics. We found the hospitals in the central of China had lower rates of poor functional outcome compared with the hospitals in the west of China, which may be partly due to the difference in economic level. In the previous study, some hospital characteristics were found to be associated with a lower risk of poor functional outcome, such as teaching hospitals and certified primary stroke centers (10). More studies designed to assess the association between hospital characteristics and outcomes at the hospital level are needed.

In this study, only $0.8 \%$ and $6.5 \%$ of the variation at 3 months and 1 year was explained by the process of care, which was less than the percentage we expected. It was presumed that the process of care affected the outcomes after stroke. A series of indicators in process of care, which were supported by evidence-based clinical guidelines, were proposed to assess and promote the quality of stroke care (27). With respect to the association between process and outcome, some studies (28-30) have found the significant association, while others $(31,32)$ have not. The uncertainty of association between process of care and outcome may result from a different selection of process of care and outcomes. The specific process of care may affect an outcome. For example, the MT procedure mainly improves functional outcomes but not mortality (33). More evidence is needed to confirm the associations between the process of care and clinical outcomes, which would help to select the target process of care to improve the outcomes.

We found medication persistence after index stroke was another source of hospital variation in functional outcomes, although it accounted for only $1.4 \%$ and $5.0 \%$ of the variation at 3 months and 1 year, respectively. Nonpersistence with secondary preventative medications is an independent factor for poor outcomes after stroke (18). There were approximately $20 \%$ of patients who failed to persist with prescribed medication at discharge in the current study. Besides education or counseling, new model and novel technology have been proposed to improve medication persistence $(34,35)$.

In the current study, the identified source of hospitallevel variation in poor functional outcome can be categorized into two groups: unmodifiable factors and modifiable factors. From the perspective of care providers, the process of care and medication persistence are the modifiable factors. Although the contribution of the process of care and medication persistence after index stroke was only $11.5 \%$ of the variation for 1 -year functional outcome, interventions targeting these processes of care and measures to improve the medication persistence should be applied to reduce the variation in functional outcomes across hospitals. For the unidentified sources of variation, we hypothesize that the care that patients received after discharge could play an important role, such as medical management of post-stroke depression, epilepsy, cognitive impairment, and access to therapy and rehabilitation specialists.

There are several limitations to the current study. First, due to the difference in patient characteristics and the process of care, this result could not be generalized into other regions or countries. The contributions of the identified factors should be confirmed by further studies. Second, most of the patients included were mild stroke patients. Although we incorporated NIHSS in the riskadjusted model to adjust for the severity of stroke, bias may exist. Thus, we should interpret the result with caution. 
Third, in this study, we analyzed the contribution of the in-hospital process of care. However, some in-hospital processes of care such as carotid surgery were not analyzed. Additionally, the covariates on post-discharge care cannot be included, which may be an important potential source of variation, especially for long-term outcomes. More research is needed to explore the contribution of post-discharge care to hospital variation in functional outcomes. Fourth, the number of patients included in some hospitals was small, which may lead to unreliable rates of poor functional outcome. To minimize this effect, we confined the included hospital to $>20$ patients.

\section{Conclusions}

In conclusion, there was hospital-level variation in functional outcome at 3 months and 1 year after acute ischemic stroke, and the patient characteristics, hospital characteristics, inhospital process of care, and medication persistence after index stroke can explain $36.8 \%$ and $46.1 \%$ of this variation. More than half of the variation is unexplained. A better understanding of the hospital variation can help to identified hospitals with higher risk and take measures to improve the functional outcomes. The extent to which the post-discharge care patients received contributes to the variation should be explored in future studies.

\section{Acknowledgments}

Funding: This study was supported by the Ministry of Science and Technology of the People's Republic of China (2018YFC1315305, 2017YFC1307702, 2018YFC1312903), Capital's Funds for Health Improvement and Research (2020-1-2041), National Natural Science Foundation of China (81870905), Beijing Municipal Science \& Technology Commission (D171100003017002).

\section{Footnote}

Reporting Checklist: The authors have completed the STROBE reporting checklist. Available at https://dx.doi. org/10.21037/apm-21-1860

Data Sharing Statement: Available at https://dx.doi. org/10.21037/apm-21-1860

Conflicts of Interest: All authors have completed the ICMJE uniform disclosure form (available at https://dx.doi. org/10.21037/apm-21-1860). The authors have no conflicts of interest to declare.

Ethical Statement: The authors are accountable for all aspects of the work in ensuring that questions related to the accuracy or integrity of any part of the work are appropriately investigated and resolved. The study was conducted in accordance with the Declaration of Helsinki (as revised in 2013). The CNSR-III study was approved by the institutional review board of Beijing Tiantan Hospital (NO. KY2015-001-01) and informed consent was taken from all the patients.

Open Access Statement: This is an Open Access article distributed in accordance with the Creative Commons Attribution-NonCommercial-NoDerivs 4.0 International License (CC BY-NC-ND 4.0), which permits the noncommercial replication and distribution of the article with the strict proviso that no changes or edits are made and the original work is properly cited (including links to both the formal publication through the relevant DOI and the license). See: https://creativecommons.org/licenses/by-nc-nd/4.0/.

\section{References}

1. GBD 2017 Causes of Death Collaborators. Global, regional, and national age-sex-specific mortality for 282 causes of death in 195 countries and territories, 1980-2017: a systematic analysis for the Global Burden of Disease Study 2017. Lancet 2018;392:1736-88.

2. Katzan IL, Spertus J, Bettger JP, et al. Risk adjustment of ischemic stroke outcomes for comparing hospital performance: a statement for healthcare professionals from the American Heart Association/American Stroke Association. Stroke 2014;45:918-44.

3. Liu S, Li Y, Zeng X, et al. Burden of Cardiovascular Diseases in China, 1990-2016: Findings From the 2016 Global Burden of Disease Study. JAMA Cardiol 2019;4:342-52.

4. GBD 2016 Stroke Collaborators. Global, regional, and national burden of stroke, 1990-2016: a systematic analysis for the Global Burden of Disease Study 2016. Lancet Neurol 2019;18:439-58.

5. Hacke W, Kaste M, Bluhmki E, et al. Thrombolysis with alteplase 3 to 4.5 hours after acute ischemic stroke. $\mathrm{N}$ Engl J Med 2008;359:1317-29.

6. Goyal M, Menon BK, van Zwam WH, et al. Endovascular thrombectomy after large-vessel ischaemic stroke: a meta- 
analysis of individual patient data from five randomised trials. Lancet 2016;387:1723-31.

7. Phipps MS, Cronin CA. Management of acute ischemic stroke. BMJ 2020;368:16983.

8. Kernan WN, Ovbiagele B, Black HR, et al. Guidelines for the prevention of stroke in patients with stroke and transient ischemic attack: a guideline for healthcare professionals from the American Heart Association/ American Stroke Association. Stroke 2014;45:2160-236.

9. Heuschmann PU, Biegler MK, Busse O, et al. Development and implementation of evidence-based indicators for measuring quality of acute stroke care: the Quality Indicator Board of the German Stroke Registers Study Group (ADSR). Stroke 2006;37:2573-8.

10. Bettger JP, Thomas L, Liang L, et al. Hospital Variation in Functional Recovery After Stroke. Circ Cardiovasc Qual Outcomes 2017;10:e002391.

11. Lingsma HF, Dippel DW, Hoeks SE, et al. Variation between hospitals in patient outcome after stroke is only partly explained by differences in quality of care: results from the Netherlands Stroke Survey. J Neurol Neurosurg Psychiatry 2008;79:888-94.

12. Wang Y, Jing J, Meng X, et al. The Third China National Stroke Registry (CNSR-III) for patients with acute ischaemic stroke or transient ischaemic attack: design, rationale and baseline patient characteristics. Stroke Vasc Neurol 2019;4:158-64.

13. Aho K, Harmsen P, Hatano S, et al. Cerebrovascular disease in the community: results of a WHO collaborative study. Bull World Health Organ 1980;58:113-30.

14. Powers WJ, Rabinstein AA, Ackerson T, et al. Guidelines for the Early Management of Patients With Acute Ischemic Stroke: 2019 Update to the 2018 Guidelines for the Early Management of Acute Ischemic Stroke: A Guideline for Healthcare Professionals From the American Heart Association/American Stroke Association. Stroke 2019;50:e344-418.

15. Fonarow GC, Reeves MJ, Smith EE, et al. Characteristics, performance measures, and in-hospital outcomes of the first one million stroke and transient ischemic attack admissions in get with the guidelines-stroke. Circ Cardiovasc Qual Outcomes 2010;3:291-302.

16. Tan Y, Pan Y, Liu L, et al. One-year outcomes and secondary prevention in patients after acute minor stroke: results from the China National Stroke Registry. Neurol Res 2017;39:484-91.

17. Kim J, Lee HS, Nam CM, et al. Effects of Statin Intensity and Adherence on the Long-Term Prognosis After Acute
Ischemic Stroke. Stroke 2017;48:2723-30.

18. Ji R, Liu G, Shen H, et al. Persistence of secondary prevention medications after acute ischemic stroke or transient ischemic attack in Chinese population: data from China National Stroke Registry. Neurol Res 2013;35:29-36.

19. Malik AO, Chhatriwalla AK, Saxon J, et al. SiteLevel Variability in 30-Day Patient Outcomes After Transcatheter Mitral Valve Repair in the United States. Circ Cardiovasc Qual Outcomes 2020;13:e006878.

20. Yeh RW, Rosenfield K, Zelevinsky K, et al. Sources of hospital variation in short-term readmission rates after percutaneous coronary intervention. Circ Cardiovasc Interv 2012;5:227-36.

21. Merlo J, Yang M, Chaix B, et al. A brief conceptual tutorial on multilevel analysis in social epidemiology: investigating contextual phenomena in different groups of people. J Epidemiol Community Health 2005;59:729-36.

22. Bushnell CD, Olson DM, Zhao X, et al. Secondary preventive medication persistence and adherence 1 year after stroke. Neurology 2011;77:1182-90.

23. O'Donnell MJ, Fang J, D'Uva C, et al. The PLAN score: a bedside prediction rule for death and severe disability following acute ischemic stroke. Arch Intern Med 2012;172:1548-56.

24. Ntaios G, Faouzi M, Ferrari J, et al. An integer-based score to predict functional outcome in acute ischemic stroke: the ASTRAL score. Neurology 2012;78:1916-22.

25. Béjot Y, Jacquin A, Daubail B, et al. Population-based validation of the iScore for predicting mortality and early functional outcome in ischemic stroke patients. Neuroepidemiology 2013;41:169-73.

26. Liu G, Ntaios G, Zheng H, et al. External validation of the ASTRAL score to predict 3- and 12-month functional outcome in the China National Stroke Registry. Stroke 2013;44:1443-5.

27. Reeves MJ, Parker C, Fonarow GC, et al. Development of stroke performance measures: definitions, methods, and current measures. Stroke 2010;41:1573-8.

28. Ido MS, Frankel MR, Okosun IS, et al. Quality of Care and Its Impact on One-Year Mortality: The Georgia Coverdell Acute Stroke Registry. Am J Med Qual 2018;33:86-92.

29. Hoenig H, Duncan PW, Horner RD, et al. Structure, process, and outcomes in stroke rehabilitation. Med Care 2002;40:1036-47.

30. Duncan PW, Horner RD, Reker DM, et al. Adherence to postacute rehabilitation guidelines is associated with 
functional recovery in stroke. Stroke 2002;33:167-77.

31. McNaughton $\mathrm{H}, \mathrm{McPherson} \mathrm{K}$, Taylor $\mathrm{W}$, et al.

Relationship between process and outcome in stroke care. Stroke 2003;34:713-7.

32. Adelman EE, Lisabeth LD, Smith MA, et al. Stroke Performance Measures Do Not Predict Functional Outcome. Neurohospitalist 2017;7:113-21.

33. McCarthy DJ, Diaz A, Sheinberg DL, et al. LongTerm Outcomes of Mechanical Thrombectomy for Stroke: A Meta-Analysis. ScientificWorldJournal

Cite this article as: Zhang R, Liu G, Pan Y, Jiang Y, Huang X, Meng X, Wang Y, Zhou M. Sources of hospital-level variation in functional outcome after acute ischemic stroke: a multicenter retrospective cohort study. Ann Palliat Med 2021;10(11):1132211332. doi: 10.21037/apm-21-1860
2019;2019:7403104.

34. Bushnell C, Arnan M, Han S. A new model for secondary prevention of stroke: transition coaching for stroke. Front Neurol 2014;5:219.

35. Zhang Y, Fan D, Ji H, et al. Treatment Adherence and Secondary Prevention of Ischemic Stroke Among Discharged Patients Using Mobile Phone- and WeChatBased Improvement Services: Cohort Study. JMIR Mhealth Uhealth 2020;8:e16496. 